\title{
Continuous subcortical motor evoked potential stimulation using the tip of an ultrasonic aspirator for the resection of motor eloquent lesions
}

\author{
Ehab Shiban, MD, Sandro M. Krieg, MD, Thomas Obermueller, MD, Maria Wostrack, MD, \\ Bernhard Meyer, MD, and Florian Ringel, MD \\ Department of Neurosurgery, Technische Universität München, Munich, Germany
}

OBJECT Resection of a motor eloquent lesion has become safer because of intraoperative neurophysiological monitoring (IOM). Stimulation of subcortical motor evoked potentials (scMEPs) is increasingly used to optimize patient safety. So far, scMEP stimulation has been performed intermittently during resection of eloquently located lesions. Authors of the present study assessed the possibility of using a resection instrument for continuous stimulation of scMEPs.

METHODS An ultrasonic surgical aspirator was attached to an IOM stimulator and was used as a monopolar subcortical stimulation probe. The effect of the aspirator's use at different ultrasound power levels $(0 \%, 25 \%, 50 \%, 75 \%$, and $100 \%$ ) on stimulation intensity was examined in a saline bath. Afterward monopolar stimulation with the surgical aspirator was used during the resection of subcortical lesions in the vicinity of the corticospinal tract in 14 patients in comparison with scMEP stimulation via a standard stimulation electrode. During resection, the stimulation current at which an MEP response was still measurable with subcortical stimulation using the surgical aspirator was compared with the corresponding stimulation current needed using a standard monopolar subcortical stimulation probe at the same location.

RESULTS The use of ultrasound at different energy levels did result in a slight but irrelevant increase in stimulation energy via the tip of the surgical aspirator in the saline bath. Stimulation of SCMEPs using the surgical aspirator or monopolar probe was successful and almost identical in all patients. One patient developed a new permanent neurological deficit. Transient new postoperative paresis was observed in $28 \%$ (4 of 14) of cases. Gross-total resection was achieved in $64 \%$ (9 of 14) cases and subtotal resection (> $80 \%$ of tumor mass) in $35 \%$ (5 of 14).

CONCLUSIONS Continuous motor mapping using subcortical stimulation via a surgical aspirator, in comparison with the sequential use of a standard monopolar stimulation probe, is a feasible and safe method without any disadvantages. Compared with the standard probe, the aspirator offers continuous information on the distance to the corticospinal tract. http://thejns.org/doi/abs/10.3171/2014.11.JNS141555

KEY WORDS intraoperative neuromonitoring; cortical stimulation; subcortical stimulation; corticospinal tract; motor eloquent tumors; diagnostic and operative techniques

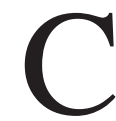
ONTINUOUs motor evoked potential (MEP) monitoring via direct cortical or transcranial stimulation is an established method of neuromonitoring during the resection of lesions in or near the corticospinal tract (CST). 3, $, 9,14,18,21,22$ Subcortical MEP (scMEP) stimulation is an additional intraoperative neurophysiological monitoring (IOM) tool, which is very helpful in assessing the proximity of the stimulation point to the CST. Thus, it supports the surgeon in determining where the resection should be stopped to avoid injury to the CST. $3,4,7,12,13,15,18$, $19,21,22,26$
However, the use of scMEP stimulation has always involved an interruption in the resection, a change to a handheld stimulation probe for mapping the resection borders. Therefore, a balance between safety (frequent halting of the resection and changing the instruments for mapping) and a continuous resective workflow (infrequent changing of the instruments for mapping) was necessary. Using a resective instrument as a stimulation probe would be ideal to enhance the workflow and safety of resections close to the CST.

In the present study we describe an approach in which

ABBREVIATIONS CST = corticospinal tract; DTI = diffusion tensor imaging; IOM = intraoperative neurophysiological monitoring; MEP = motor evoked potential; scMEP = subcortical MEP.

SUBMITTED July 3, 2014. ACCEPTED November 5, 2014.

INCLUDE WHEN CITING Published online May 15, 2015; DOI: 10.3171/2014.11.JNS141555.

DISCLOSURE The authors report no conflict of interest concerning the materials or methods used in this study or the findings specified in this paper. Dr. Krieg is a consultant for BrainLAB AG. 
the tip of a surgical aspirator was evaluated for its utility as an scMEP stimulation probe that would allow continuous stimulation and parallel resection with the same surgical instrument.

\section{Methods}

The institutional review board approved all study protocols, and written informed consent was obtained from each patient before participating in the study.

\section{Setup for scMEP Stimulation}

An ultrasonic surgical aspirator (SonaStar ultrasonic surgical aspiration system, Misonix Inc.) was used for tumor resection and as a monopolar stimulation probe for scMEPs. The electrical stimulator of a monitoring system (ISIS IOM neuromonitoring system, Inomed Medizintechnik) was directly connected to the electrosurgery monopolar radiofrequency connector of the handpiece of the ultrasonic aspirator.

\section{Effect of Surgical Aspirator on Electrical Stimulation}

To assess the influence of ultrasound energy from the surgical aspirator on monopolar stimulation from the tip of the aspirator, we performed a bench experiment. The ultrasonic aspirator was connected to the IOM stimulator, and the tip of the aspirator was placed in a $0.9 \%$ saline bath, which was supplied with a ground electrode as a second electrode for stimulation and with 2 electrodes for voltage detection, as shown in Fig. 1. Stimulation (delivered as cathodal stimulation) conditions were as follows: square-wave pulses with a duration of $0.3 \mathrm{msec}$ and a train of 5 pulses and current intensity of $3 \mathrm{~mA}$.

Stimulation was initially performed without activation of the ultrasound, and a train of five impulses and the respective curves were documented for evaluation. Subsequently, the effects of different ultrasound activation levels $(25 \%, 50 \%, 75 \%$, and $100 \%$ of maximum ultrasound intensity) on the resulting current in the saline bath were measured for 5 stimulation impulses, each according to the train-of-five technique. ${ }^{25}$ This sequence was per-

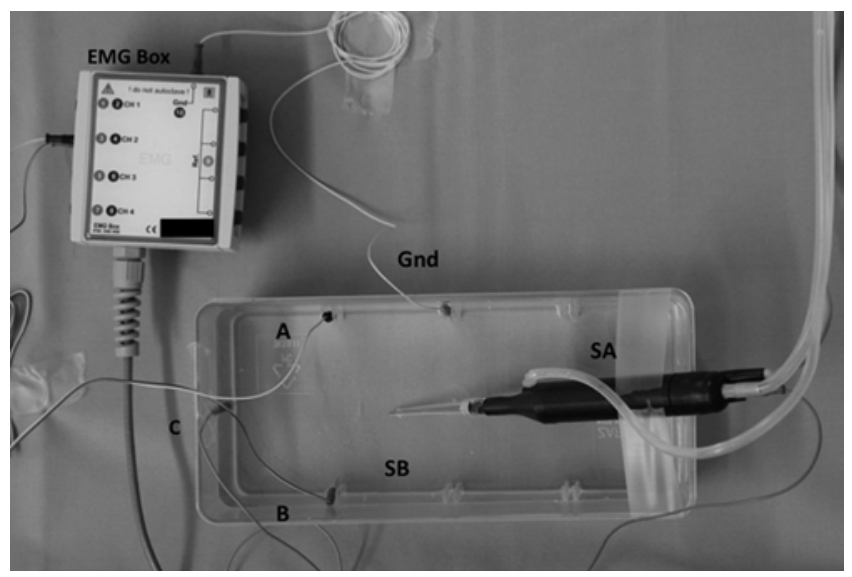

FIG. 1. The ultrasonic surgical aspirator (SA) is connected to an IOM stimulator through an adapter cable. Electrical stimulation voltage is detected through the needles $(A$ and $B)$ in a saline bath (SB). Note the anodal pole for the monopolar stimulation $(C)$ and ground electrode (Gnd). EMG = electromyography. formed 3 times, and the average of these stimulations was calculated and compared with the stimulation intensity at baseline (without surgical aspirator activation).

\section{Patient Cohort}

Between August 2013 and December 2013, in 14 patients with motor eloquent lesions surgically resected in our department, stimulation of scMEPs using a standard monopolar stimulation probe was compared with that delivered through an aspirator tip at the end of resection. Patient characteristics are summarized in Table 1. Motor eloquence was defined as $<2 \mathrm{~cm}$ of distance between the lesion and the CST, as depicted by preoperative diffusion tensor imaging (DTI) of motor fiber tracking (Fig. 2). Intraoperative continuous MEP monitoring via cortical stimulation was performed in all cases and was supplemented by subcortical motor stimulation in all cases. An image guidance system was also used in all cases (VectorVision2, BrainLAB). Neurological examination was performed in every patient preoperatively, directly after surgery, at discharge 5-10 days after surgery, and at the first follow-up 3 months after surgery. Motor function was graded according to the British Medical Research Council (MRC) Scale.

Magnetic resonance imaging was acquired before and within 48 hours after surgery in all cases. A whole-body 3-T imaging system (Achieva, Philips Electronics) with an 8-channel head coil was used. Three-dimensional imaging was performed through continuous sagittal images using a T1-weighted 3D gradient echo sequence with isotropic voxels of $1 \mathrm{~mm}$ in length after the intravenous administration of $0.1 \mathrm{mmol} / \mathrm{kg}$ of gadopentetate dimeglumine. The 3D data sets were transferred to the neuronavigation planning system (iPlan Cranial 3.0.1, BrainLAB AG) and fused with T2 FLAIR and DTI data.

\section{Neurophysiological Monitoring}

Continuous cortical stimulation with the train-of-five method was used with the Inomed ISIS IOM system, as previously described. ${ }^{11,22}$ During tumor resection, MEPs were elicited every 1-15 seconds depending on the location of resection. Subcortical stimulation with the trainof-five method was delivered using either a concentric monopolar handheld probe (Inomed Medizintechnik), as previously described ${ }^{22}$ or the tip of the ultrasonic surgical aspirator.

Upon approaching approximately $20 \mathrm{~mm}$ of distance from the CST, as depicted by the neuronavigation system, subcortical stimulation was started. Stimulation intensity was initially set at $20 \mathrm{~mA}$ with a $300-\mathrm{msec}$ pulse duration at $60 \mathrm{~Hz}$. Tumor removal was continued using simultaneous resection and stimulation until an MEP was elicited. Thereafter, stimulation intensity was reduced in 4-mA steps until stimulation intensities were below $8 \mathrm{~mA}$. Subsequently, stimulation intensity reduction occurred in 1-mA steps. Resection was stopped at stimulation intensities of $3 \mathrm{~mA}$. The scMEP stimulation thresholds were compared at the same points within the resection cavity using stimulation with either the monopolar probe or the surgical aspirator tip, and agreement was analyzed. 
TABLE 1. Summary of characteristics in 14 patients with lesions in motor eloquent regions

\begin{tabular}{|c|c|c|c|c|c|c|c|}
\hline \multirow{2}{*}{$\begin{array}{c}\text { Case } \\
\text { No. }\end{array}$} & \multirow{2}{*}{$\begin{array}{l}\text { Age } \\
\text { (yrs) }\end{array}$} & \multirow[b]{2}{*}{ Sex } & \multirow[b]{2}{*}{ Pathology } & \multirow[b]{2}{*}{ cMEPs } & \multicolumn{2}{|c|}{ Subcortical Stimulation Motor Threshold (mA) } & \multirow[b]{2}{*}{ New Postop Motor Deficit } \\
\hline & & & & & MP & SA & \\
\hline 1 & 43 & M & Met & Stable & 9 & 9 & No \\
\hline 2 & 62 & M & GBM & Stable & 18 & 18 & No \\
\hline 3 & 40 & M & AS II & Permanent loss & 5 & 6 & Temporary \\
\hline 4 & 52 & M & $\mathrm{AO}$ & Stable & 4 & 4 & Temporary \\
\hline 5 & 43 & $\mathrm{~F}$ & GBM & Permanent loss & $10 ; 10 ; 11$ & $9 ; 10 ; 11$ & Permanent \\
\hline 6 & 55 & $\mathrm{M}$ & GBM & Stable & 7 & 8 & Temporary \\
\hline 7 & 43 & $F$ & AS II & Stable & 9 & 9 & No \\
\hline 8 & 55 & $\mathrm{M}$ & GBM & Stable & 3 & 3 & No \\
\hline 9 & 69 & M & Met & Stable & $6 ; 3$ & $7 ; 3$ & No \\
\hline 10 & 58 & M & GBM & Stable & 12 & 12 & No \\
\hline 11 & 32 & $\mathrm{~F}$ & Met & Stable & 17 & 17 & No \\
\hline 12 & 78 & $\mathrm{~F}$ & GBM & Stable & 6 & 6 & No \\
\hline 13 & 60 & $F$ & GBM & Temporary loss & 6 & 5 & Temporary \\
\hline 14 & 57 & $\mathrm{~F}$ & Met & Stable & 4 & 4 & No \\
\hline
\end{tabular}

$\mathrm{AO}$ = anaplastic oligodendroglioma; $\mathrm{AS} \|$ = astrocytoma Grade II; cMEP = cortical motor evoked potential, GBM = glioblastoma multiforme; Met $=$ metastasis; $\mathrm{MP}=$ monopolar probe $; \mathrm{SA}=$ surgical aspirator .

\section{Statistical Analyses}

Agreement between both stimulation methods was analyzed using a Bland-Altman plot and correlations. Statistical analyses were performed using SPSS for Mac, version 22 (SPSS Inc.). A p value $\leq 0.05$ was considered significant.

\section{Results}

\section{Influence of the Surgical Aspirator on Monopolar Stimulation}

Activation of the surgical aspirator increased stimulation intensity to (mean \pm standard deviation) $105.7 \% \pm$ $7.9 \%$ at the $25 \%$ aspirator activation level, $105.8 \% \pm 3.6 \%$ at the $50 \%$ level, $106.5 \% \pm 8.1 \%$ at the $75 \%$ level, and $106.9 \% \pm 13.9 \%$ at the $100 \%$ level compared with baseline (without surgical aspirator activation; Fig. 3).

\section{Patient Characteristics}

Fourteen patients ( 6 females and 8 males) with motor eloquent lesions underwent resection within the study period. The mean age of patients at the time of surgery was 53 years (range 32-78 years). Pathologies included 10 gliomas (7 WHO Grade IV, 1 WHO Grade III, 2 WHO Grade II) and 4 metastases. Symptoms at presentation were hemiparesis in 9 patients, headaches in 2, and aphasia in 1; 2 patients were asymptomatic. The median preoperative Karnofsky Performance Scale (KPS) score was 80 (range 70-100).

Tumor resection was performed according to microsurgical standards. Gross-total resection was achieved in $64 \%$ (9 of 14) of cases and subtotal resection $(>80 \%$ of tumor mass) in $36 \%$ (5 of 14) of cases. Among the subtotal resection cases, 3 patients had a multifocal GBM and complete resection was not possible. Upon any instability of MEP signals or amplitude decline or loss from continuous cortical stimulation, resection was stopped, spatulas were removed, and the surgical field was irrigated with warm Ringer's solution. Resection boundaries were determined using both cortical and scMEP stimulation. In most cases, resection was prematurely stopped if cortical MEP amplitudes were reduced below $50 \%$ of their initial height and/or at an scMEP threshold of $3 \mathrm{~mA}$.

\section{Correlation Between Subcortical Stimulation With the Surgical Aspirator and the Monopolar Handheld Probe}

Subcortical stimulations delivered with the handheld probe and the surgical aspirator were successful in all cases. Stimulation intensities for both methods were almost identical for electing the lowest motor threshold from the same stimulation site in all cases (Fig. 4; $\mathrm{r}^{2}=0.994$, $\mathrm{p}<$ $0.0001)$. A $1-\mathrm{mA}$ difference between both stimulation devices was regarded as clinically insignificant.

\section{Correlation Between Electrophysiological Findings and Postoperative Clinical Outcome}

The scMEP threshold ranged from 3 to $18 \mathrm{~mA}$ in both stimulation modalities. One patient (Case 5) suffered a high-grade paresis following surgery. In that case, cortical MEPs were permanently lost. Transient new postoperative paresis or temporary exacerbation of preoperative paresis was observed in $28 \%$ (4 of 14) of cases (Table 1). In all of these cases, the motor threshold was a 4- to 8-mA stimulation intensity at a $300-\mathrm{msec}$ pulse duration.

\section{Discussion}

Herein, we introduce a novel method of continuous subcortical stimulation during resection of motor eloquent lesions. Our data show that utilizing a surgical aspirator to stimulate scMEPs is feasible, safe, and virtually identical to using the standard probe with regard to motor thresholds. The effects of ultrasound energy from the surgical aspirator on stimulation intensity are negligible. 

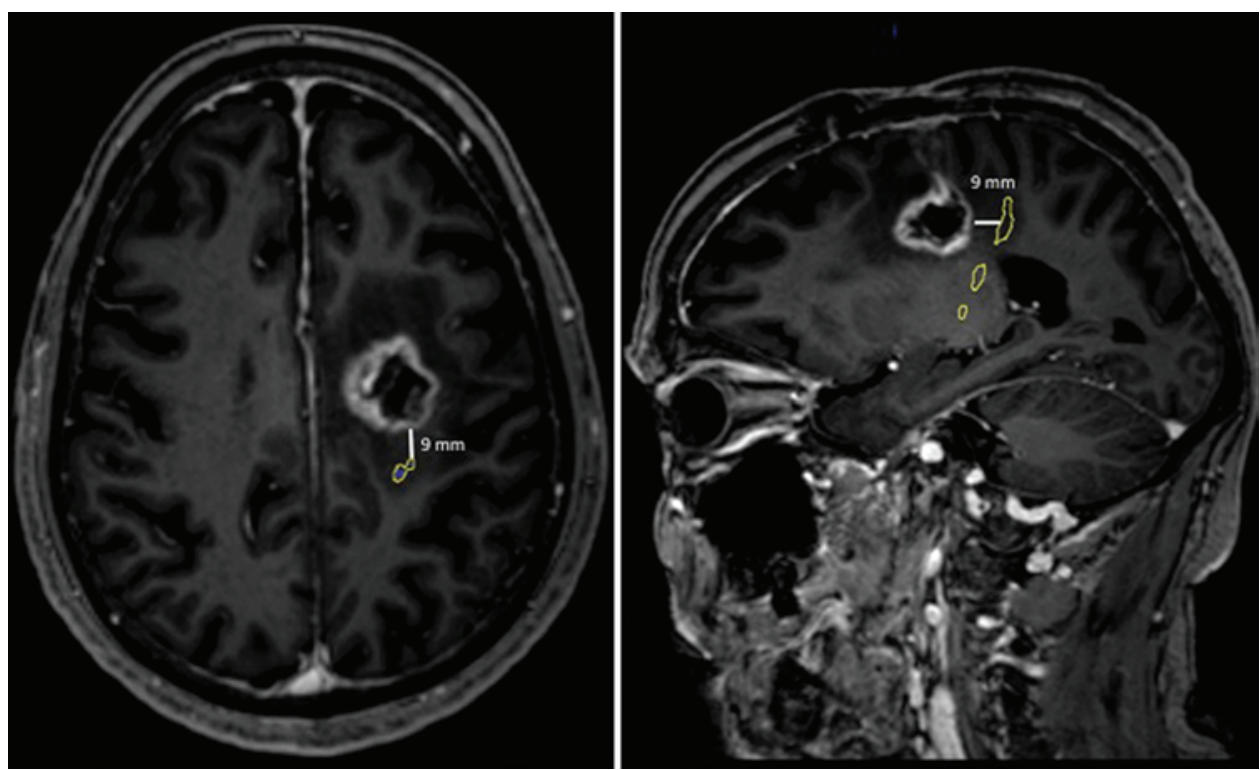

FIG. 2. Preoperative axial (left) and sagittal (right) MR images showing the distance between the CST (yellow) and the lesion (white line). Figure is available in color online only.

There is growing evidence and agreement that the extent of resection correlates with patient survival. This holds true for both high-grade 20,23 and low-grade gliomas. ${ }^{2,5}$ Moreover, for glioblastoma, there is even some evidence suggesting that gross-total resection is most benefi-

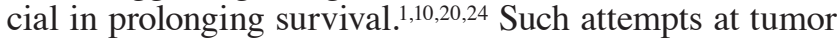

removal should be pursued only when the basic principle of avoiding new permanent neurological deficits is possible to follow. To prevent permanent deficits and to increase the extent of resection, different methods of intraoperative mapping and monitoring of neurological functions are used.

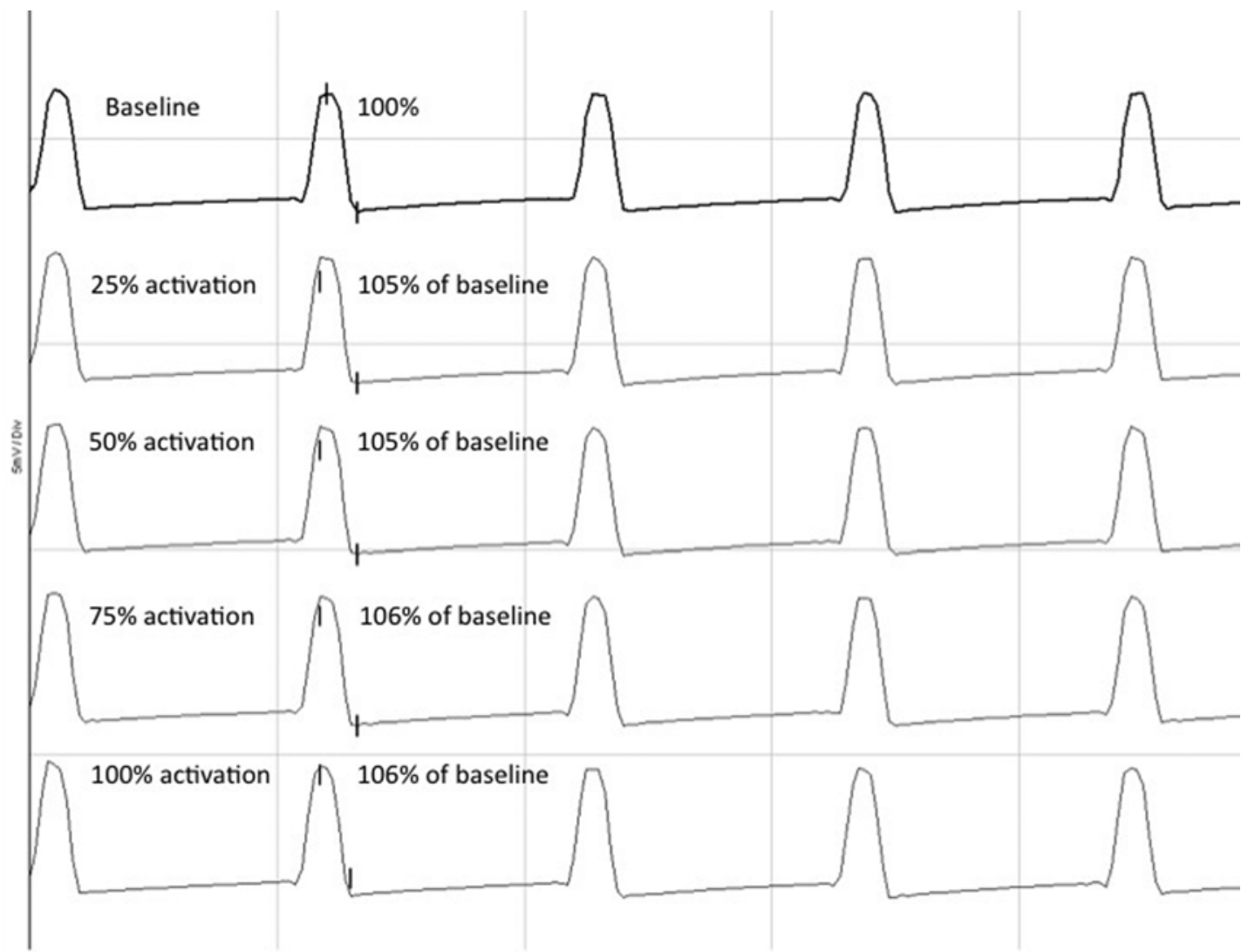

FIG. 3. Influence of surgical aspirator activation on electrical stimulation voltage in the saline bath. Div = division. 


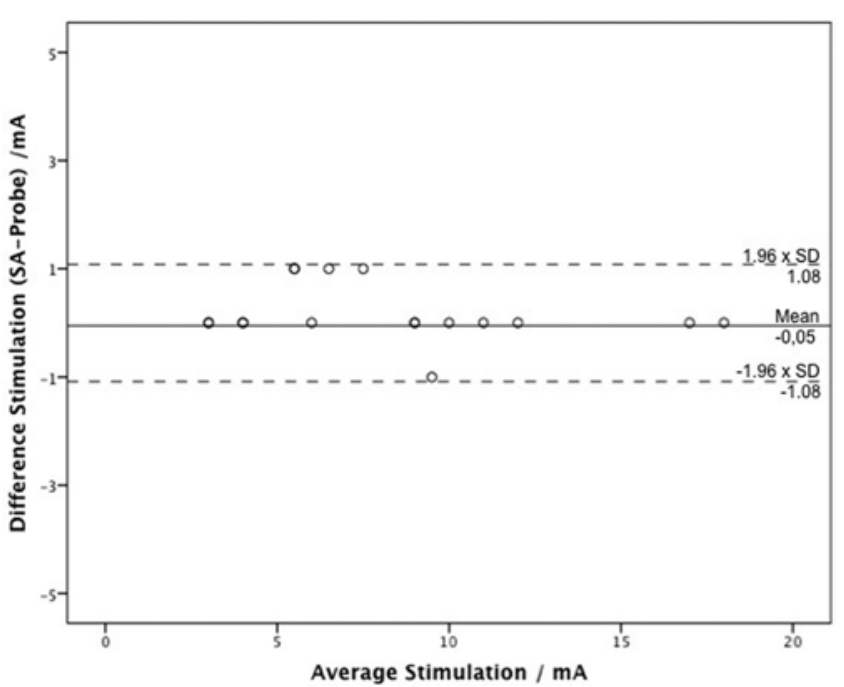

FIG. 4. Bland-Altman plot illustrating motor mapping thresholds measured with the handheld monopolar probe and surgical aspirator (SA). The variability of the different methods was evaluated and visualized on the Bland-Altman plot. The limits of agreement during Bland-Altman analysis were the average difference $\pm 1.96 \mathrm{SD}$ of the difference.

To date, subcortical stimulation of motor tracts during resection of motor eloquent lesions is gaining popularity because it allows one to estimate the location and distance of the CST and therefore reduce the risk of motor tract injury. $7,12,13,19,21$ Until recently, the spatial and temporal stimulation capabilities of scMEPs were limited since the resection had to be stopped to apply stimulation with a handheld probe in the resection cavity, thereby estimating the location and distance to the CST.6,15,16,17,18,21,22 This interruption of surgical flow is not only inconvenient and time consuming but may also increase the risk of mechanical injury to the CST. ${ }^{18,21}$ The increased risk is attributable to the fact that at presumably safe distances from the CST, resection is mostly guided by cortical MEPs. As long as cortical MEPs are stable, resection is continued without stopping the resection every $1-2 \mathrm{~mm}$ to verify the distance to the CST by scMEPs. However, because most MEP alterations from direct cortical stimulation are irreversible and occur at presumably safe distances from the CST, 18,21 the need for higher spatial and temporal scMEP capabilities became clear.

Recently, there was introduced a new device for continuous dynamic mapping-meaning continuous subcortical stimulation throughout resection. ${ }^{18}$ This device was created by connecting a modified suction device to a stimulator. The surface of the suction device was isolated to limit electrical contact with the tip of the device. The authors compared the motor mapping thresholds for stimulation sites simultaneously mapped with the scMEP and the new device in 24 patients. They found a virtual 1:1 correlation between the 2 probes.

In the present study we connected a commercially available surgical aspirator to a commercially available intraoperative stimulator. There was no need for the development of any new hardware or software. First, we studied the effects of different surgical aspirator activation levels on stimulation intensity in a bench setup. We found practically negligible differences in the stimulation intensity upon activation of the ultrasound. There were no relevant discrepancies in stimulation intensities between all 4 activation levels examined $(25 \%, 50 \%, 75 \%$, and $100 \%)$. Second, we compared the monopolar stimulation probe with the surgical aspirator stimulation during resection of the motor eloquent lesions. The motor threshold for stimulation sites simultaneously mapped with the monopolar probe and the surgical aspirator showed no relevant discrepancies. A 1-mA difference in stimulation intensity was regarded as irrelevant because upon stimulation with both modalities a slight hand movement alters the stimulation trajectory and, through that, the elicited MEP response as well. These kinds of movements are normal upon stimulation with the standard stimulation probe and have no influence on decision making during surgery.

Four patients developed a temporary new motor deficit. The motor threshold ranged from 4 to $8 \mathrm{~mA}$ in these patients (Table 1). Others have also described a "safe corridor" of tumor resection with motor thresholds between 5 and $20 \mathrm{~mA}$ in which mechanical damage to the CST and permanent motor deficit can mostly be excluded. . $, 13,15,16,18,21,22 \mathrm{~A}$ higher rate of permanent motor deficit is observed at a motor threshold below $3 \mathrm{~mA}$; therefore some recommend terminating resection at a motor threshold of $2 \mathrm{~mA}^{18,21}$ or $3 \mathrm{~mA} .^{.22}$

One patient (Case 5) suffered a permanent, new motor deficit; she had a very large multifocal glioblastoma and presented with slight contralateral hemiparesis. The motor threshold in this case was obtained during resection at 3 separate stages and was virtually identical upon comparing the 2 stimulation modalities (Table 1). Cortical MEPs started to deteriorate and were eventually lost during the final resection stage. Postoperative MRI revealed ischemia of the CST at the mesial part of the resection cavity.

\section{Conclusions}

Continuous motor mapping using subcortical stimulation via the tip of an ultrasonic aspirator is a feasible and safe method of intraoperative mapping. Utility and electrical distribution are practically identical to those in standard stimulation techniques using the handheld stimulation probe. Whether continuous subcortical stimulation has an additional benefit for patients is still unclear and can only be truly answered with a comparative study.

\section{References}

1. Chaichana KL, Zadnik P, Weingart JD, Olivi A, Gallia GL, Blakeley J, et al: Multiple resections for patients with glioblastoma: prolonging survival. J Neurosurg 118:812-820, 2013

2. Chang EF, Clark A, Smith JS, Polley MY, Chang SM, Barbaro NM, et al: Functional mapping-guided resection of low-grade gliomas in eloquent areas of the brain: improvement of long-term survival. Clinical article. J Neurosurg 114:566-573, 2011

3. De Witt Hamer PC, Robles SG, Zwinderman AH, Duffau $\mathrm{H}$, Berger MS: Impact of intraoperative stimulation brain mapping on glioma surgery outcome: a meta-analysis. J Clin Oncol 30:2559-2565, 2012

4. Duffau H: Lessons from brain mapping in surgery for low- 
grade glioma: insights into associations between tumour and brain plasticity. Lancet Neurol 4:476-486, 2005

5. Jakola AS, Myrmel KS, Kloster R, Torp SH, Lindal S, Unsgård G, et al: Comparison of a strategy favoring early surgical resection vs a strategy favoring watchful waiting in lowgrade gliomas. JAMA 308:1881-1888, 2012

6. Kamada K, Todo T, Ota T, Ino K, Masutani Y, Aoki S, et al: The motor-evoked potential threshold evaluated by tractography and electrical stimulation. J Neurosurg 111:785-795, 2009

7. Keles GE, Lundin DA, Lamborn KR, Chang EF, Ojemann G, Berger MS: Intraoperative subcortical stimulation mapping for hemispherical perirolandic gliomas located within or adjacent to the descending motor pathways: evaluation of morbidity and assessment of functional outcome in 294 patients. J Neurosurg 100:369-375, 2004

8. Kombos T, Picht T, Derdilopoulos A, Suess O: Impact of intraoperative neurophysiological monitoring on surgery of high-grade gliomas. J Clin Neurophysiol 26:422-425, 2009

9. Kombos T, Suess O, Ciklatekerlio O, Brock M: Monitoring of intraoperative motor evoked potentials to increase the safety of surgery in and around the motor cortex. J Neurosurg 95:608-614, 2001

10. Kreth FW, Thon N, Simon M, Westphal M, Schackert G, Nikkhah G, et al: Gross total but not incomplete resection of glioblastoma prolongs survival in the era of radiochemotherapy. Ann Oncol 24:3117-3123, 2013

11. Krieg SM, Shiban E, Droese D, Gempt J, Buchmann N, Pape $\mathrm{H}$, et al: Predictive value and safety of intraoperative neurophysiological monitoring with motor evoked potentials in glioma surgery. Neurosurgery 70:1060-1071, 2012

12. Mikuni N, Okada T, Enatsu R, Miki Y, Hanakawa T, Urayama S, et al: Clinical impact of integrated functional neuronavigation and subcortical electrical stimulation to preserve motor function during resection of brain tumors. J Neurosurg 106:593-598, 2007

13. Mikuni N, Okada T, Nishida N, Taki J, Enatsu R, Ikeda A, et al: Comparison between motor evoked potential recording and fiber tracking for estimating pyramidal tracts near brain tumors. J Neurosurg 106:128-133, 2007

14. Neuloh G, Pechstein U, Schramm J: Motor tract monitoring during insular glioma surgery. J Neurosurg 106:582-592, 2007

15. Nossek E, Korn A, Shahar T, Kanner AA, Yaffe H, Marcovici $\mathrm{D}$, et al: Intraoperative mapping and monitoring of the corticospinal tracts with neurophysiological assessment and 3-dimensional ultrasonography-based navigation. Clinical article. J Neurosurg 114:738-746, 2011

16. Ohue S, Kohno S, Inoue A, Yamashita D, Harada H, Kumon $Y$, et al: Accuracy of diffusion tensor magnetic resonance imaging-based tractography for surgery of gliomas near the pyramidal tract: a significant correlation between subcortical electrical stimulation and postoperative tractography. Neurosurgery 70:283-294, 2012

17. Prabhu SS, Gasco J, Tummala S, Weinberg JS, Rao G: Intraoperative magnetic resonance imaging-guided tractography with integrated monopolar subcortical functional mapping for resection of brain tumors. Clinical article. J Neurosurg 114:719-726, 2011

18. Raabe A, Beck J, Schucht P, Seidel K: Continuous dynamic mapping of the corticospinal tract during surgery of motor eloquent brain tumors: evaluation of a new method. J Neurosurg 120:1015-1024, 2014

19. Sanai N, Berger MS: Intraoperative stimulation techniques for functional pathway preservation and glioma resection. Neurosurg Focus 28(2):E1, 2010

20. Sanai N, Polley MY, McDermott MW, Parsa AT, Berger MS: An extent of resection threshold for newly diagnosed glioblastomas. J Neurosurg 115:3-8, 2011

21. Seidel K, Beck J, Stieglitz L, Schucht P, Raabe A: The warning-sign hierarchy between quantitative subcortical motor mapping and continuous motor evoked potential monitoring during resection of supratentorial brain tumors. J Neurosurg 118:287-296, 2013

22. Shiban E, Krieg SM, Haller B, Buchmann N, Obermueller $\mathrm{T}$, Boeckh-Behrens T, et al: Intraoperative subcortical motor evoked potential stimulation: how close is the corticospinal tract? J Neurosurg [in press], 2015

23. Stummer W, Pichlmeier U, Meinel T, Wiestler OD, Zanella F, Reulen HJ: Fluorescence-guided surgery with 5-aminolevulinic acid for resection of malignant glioma: a randomised controlled multicentre phase III trial. Lancet Oncol 7:392401, 2006

24. Stummer W, Reulen HJ, Meinel T, Pichlmeier U, Schumacher W, Tonn JC, et al: Extent of resection and survival in glioblastoma multiforme: identification of and adjustment for bias. Neurosurgery 62:564-576, 2008

25. Taniguchi M, Cedzich C, Schramm J: Modification of cortical stimulation for motor evoked potentials under general anesthesia: technical description. Neurosurgery 32:219-226, 1993

26. Vassal F, Schneider F, Nuti C: Intraoperative use of diffusion tensor imaging-based tractography for resection of gliomas located near the pyramidal tract: comparison with subcortical stimulation mapping and contribution to surgical outcomes. Br J Neurosurg 27:668-675, 2013

\section{Author Contributions}

Conception and design: Ringel, Shiban. Acquisition of data: Ringel, Shiban. Analysis and interpretation of data: Ringel, Shiban. Drafting the article: Shiban. Critically revising the article: Ringel, Krieg, Meyer. Reviewed submitted version of manuscript: Ringel, Obermueller, Wostrack, Meyer. Statistical analysis: Ringel, Shiban. Study supervision: Ringel.

\section{Supplemental Information}

\section{Previous Presentation}

Portions of this work have been presented in abstract form at the European Association of Neurosurgical Societies Congress held in Prague, Czech Republic, on October 12-17, 2014.

\section{Correspondence}

Florian Ringel, Department of Neurosurgery, Klinikum rechts der Isar, Technische Universität München, Ismaninger Str. 22, 81675 Munich, Germany. email: florian.ringel@lrz.tum.de. 